Conclusion We have not seen any difference in outcome for hypothermic babies, but the hypothermia rate and the rates of the adverse outcomes were low, so we cannot exclude an effect.

We have seen an increase in the rate of major cranial ultrasound abnormality in hyperthermic babies. This has been demonstrated in previous studies.

We will continue to strive to avoid hypothermia and tolerate the resulting increase in hyperthermia rate until we know whether the association between hyperthermia and brain injury is a causal association, or just an association through the shared aetiology of infection. We are undertaking further work to investigate this.

\section{G569(P) THE CORRELATION BETWEEN EARLY GENERAL MOVEMENT ASSESSMENT AND TWO YEAR NEURODEVELOPMENTAL OUTCOME IN HIGH RISK PRETERM INFANTS}

'D Paris, ${ }^{2} \mathrm{~A}$ Allman. ${ }^{1}$ Physiotherapy, Aneurin Bevan University Hospital Board, Newport, UKi ${ }^{2}$ Neonatalolgy, Aneurin Bevan University Hospital Board, Newport, UK

\subsection{6/archdischild-2020-rcpch.487}

Aims The Prechtl General Movement Assessment (GMA) is a reliable tool for identifying babies at risk of neuromotor deficit. We sought to compare our GMA results with standardised Bayley's III neurodevelopmental outcomes for a population of high risk babies at a corrected age of 2 years.

Methods High risk premature Infants born during 2016 who received their neonatal intensive care in a level 3 NICU were retrieved from neonatal databases. Those infants who had GMA performed were extracted and the GMA results were compared with the results of 2 year standardised neurodevelopmental assessments.

Results In 2016, 32 babies born between 23 and 32 weeks gestation had GMA performed between 10 to 15 weeks of age. Most infants had 2 assessments. Their birth weights ranged from $570 \mathrm{~g}$ to 1850 g. 2 babies were lost to further follow up. 30 were invited for Bayley's III neurodevelopmental assessment at a corrected age of 2 years and of these 3 are still awaiting assessment. Of the 27 assessed, 22 $(82 \%)$ have a normal 2 year outcome and $21(95 \%)$ of the 22 had normal GMA. One had equivocal GMA. 2(7\%) of the 27 babies had unequivocal absent fidgety movements on GMA and both of these infants have severe or moderate delay across all Bayley's domains. 1(4\%) infant had equivocally absent fidgety movements and did not attend their Bayley assessment but have documented motor delay in standard clinic assessment. 2(7\%) infants had moderate cognitive delay on Bayley's testing, but normal motor and speech development and both of these infants had a normal GMA.

Conclusion Our study demonstrates a high level of correlation between abnormal GMA and disability as assessed by standardised methods at 2 years. This is most linked with motor dysfunction rather than cognitive. Our numbers are currently small as the GMA service is new to us, but preliminary results are encouraging. Abnormal GMA identified in the first weeks of life can be used to target those babies for enhanced intervention thus improving their final outcome.

\section{G570(P) THE EFFECT OF INTRAUTERINE GROWTH RETARDATION ON THE LUNG FUNCTION OF PREMATURELY BORN YOUNG PEOPLE}

${ }^{1} \mathrm{C}$ Harris, 'A Lunt, ${ }^{2} \mathrm{~J}$ Peacock, 'A Greenough. 'School of Life Course Sciences, King's College London, London, UKi ${ }^{2}$ Primary Care and Public Health Sciences, King's College London, London, UK

\subsection{6/archdischild-2020-rcpch.488}

Background Poor intrauterine growth has been shown to result in increased morbidity in infancy. Our aim was to examine the effect of intrauterine growth retardation on the lung function of prematurely born young people routinely exposed to antenatal steroids and postnatal surfactant.

Methods Lung function was assessed at 16-19 years of age. Forced expired volume at 1 second $\left(\mathrm{FEV}_{1}\right)$, forced vital capacity (FVC), forced expiratory flow at $75 \%\left(\mathrm{FEF}_{75}\right)$, lung volumes by plethysmography, diffusion capacity of the lungs for carbon monoxide $\left(\mathrm{D}_{\mathrm{L}} \mathrm{CO} / \mathrm{VA}\right)$ measurements were taken and converted to $\mathrm{z}$ scores based on sex and height. A birthweight $<$ the 10 th centile was considered small for gestational age (SGA) and those with greater birth weight appropriate for gestational age (AGA).

Results One hundred and fifty-eight young people were assessed, 37 were SGA:

\begin{tabular}{|c|c|c|c|}
\hline & $\begin{array}{l}\text { AGA } \\
\text { Mean (SD) } \\
\text { or } N(\%)\end{array}$ & $\begin{array}{l}\text { SGA } \\
\text { Mean (SD) } \\
\text { or } N(\%)\end{array}$ & $P$ value \\
\hline Male & $63(52)$ & $19(51)$ & 0.939 \\
\hline Gestational age (wks) & $26.8(1.50)$ & $27.2(1.20)$ & 0.085 \\
\hline Birthweight z score & $-0.14(0.66)$ & $-2.05(0.55)$ & $<0.001$ \\
\hline Postnatal steroids & $32(26)$ & $16(43)$ & 0.052 \\
\hline BPD & $60(50)$ & $25(68)$ & 0.055 \\
\hline At assessment age (yrs) & $17.4(0.84)$ & $17.5(0.73)$ & 0.888 \\
\hline Height $(\mathrm{cm})$ & $168(8.89)$ & $164(9.13)$ & 0.027 \\
\hline Weight (kg) & $65(15.7)$ & $56(15.8)$ & 0.006 \\
\hline $\mathrm{FEV}_{1}$ & $-0.87(1.27)$ & $-1.43(1.40)$ & 0.028 \\
\hline FVC & $-0.13(1.33)$ & $-0.57(1.34)$ & 0.093 \\
\hline $\mathrm{FEV}_{1}$ :FVC & $-1.06(1.19)$ & $-1.36(1.27)$ & 0.203 \\
\hline $\mathrm{FEF}_{75}$ & $-0.89(1.25)$ & $-1.39(1.27)$ & 0.044 \\
\hline $\mathrm{FRC}_{\text {pleth }}$ & $0.45(1.26)$ & $1.22(1.55)$ & 0.003 \\
\hline $\mathrm{RV}_{\text {pleth }}$ & $0.87(1.20)$ & $1.78(1.62)$ & $<0.001$ \\
\hline $\mathrm{TLC}_{\text {pleth }}$ & $0.76(1.17)$ & $1.16(1.15)$ & 0.079 \\
\hline $\mathrm{D}_{\mathrm{L}} \mathrm{CO} / \mathrm{VA}$ & $-1.98(1.12)$ & $-2.48(0.67)$ & 0.004 \\
\hline
\end{tabular}

Conclusion Intrauterine growth retardation was associated with impaired airway and alveolar function at 16-19 years.

\section{G571(P) NEONATAL HAEMATOCRIT MEASUREMENT: DIFFERENT METHODS, DIFFERENT RESULTS?}

E Tsouana, J Arumugam, P Nousiou, A Gupta. Department of Paediatrics, Basildon Hospital, Basildon, UK

\subsection{6/archdischild-2020-rcpch.489}

Aim Three different methods are widely available in neonatal units for the measurement of neonatal Haematocrit (HCT); centrifugation (spun micro-haematocrit; SHCT), automated 